\title{
Early Prevention Method for Power System Instability
}

Dmitrova, Evgenia; Wittrock, Martin Lindholm; Jóhannsson, Hjörtur; Nielsen, Arne Hejde

Published in:

IEEE Transactions on Power Systems

Link to article, DOI:

10.1109/TPWRS.2014.2353693

Publication date:

2015

Document Version

Publisher's PDF, also known as Version of record

Link back to DTU Orbit

Citation (APA):

Dmitrova, E., Wittrock, M. L., Jóhannsson, H., \& Nielsen, A. H. (2015). Early Prevention Method for Power System Instability. IEEE Transactions on Power Systems, 30(4), 1784-1792.

https://doi.org/10.1109/TPWRS.2014.2353693

\section{General rights}

Copyright and moral rights for the publications made accessible in the public portal are retained by the authors and/or other copyright owners and it is a condition of accessing publications that users recognise and abide by the legal requirements associated with these rights.

- Users may download and print one copy of any publication from the public portal for the purpose of private study or research.

- You may not further distribute the material or use it for any profit-making activity or commercial gain

- You may freely distribute the URL identifying the publication in the public portal

If you believe that this document breaches copyright please contact us providing details, and we will remove access to the work immediately and investigate your claim 


\title{
Early Prevention Method for Power System Instability
}

\author{
Evgenia Dmitrova, Martin Lindholm Wittrock, Student Member, IEEE, Hjörtur Jóhannsson, Member, IEEE, and
} Arne Hejde Nielsen, Senior Member, IEEE

\begin{abstract}
This paper presents a method to determine suitable countermeasures against emerging aperiodic small disturbance rotor angle (ASD) instability. The method utilizes stability indicators, computed in real time, to define a criterion for ASD stability. Sensitivities of these stability indicators are then determined and used to identify the most influential nodes for countermeasures. To increase the computational speed, only nodes visited by a self-propagating graph, rooted at the vulnerable generator, will have their sensitivities calculated. The steady state voltages after a given countermeasure are then determined, using a grid transformation coefficient (GTC) and a numerical, iterative solution to an equation system. The stability criteria can then be assessed to evaluate the sufficiency of a suggested countermeasure. The method is demonstrated on a synthetic 8-bus network and a 464-bus model of the Western Denmark transmission grid. The method successfully demonstrates its ability to efficiently identify and evaluate countermeasures for a large, practical system.
\end{abstract}

Index Terms-Power system control, power system security, power system simulation, power system stability.

\section{INTRODUCTION}

$\mathbf{T}$ HE series of blackouts experienced in North America and Europe in 2003 [1]-[4] demonstrated the need for new advanced control algorithms to be developed if safe and reliable power system operation is to be ensured in the future. Traditionally, supplementary control systems have been employed to counter stability problems, most notably controlled system separation and load shedding schemes [5]. But as seen in 2003, the size of the region affected by power system stability mechanisms is increasing just as the complexity of the involved mechanisms. For this reason investigation committees have recommended the development of practical applications for wide area monitoring and control systems using real time synchrophasor measurements [1]-[3].

These recommendations are linked with the increasing share of renewable energy sources which are being introduced to the power system. For instance, the Danish Transmission System Operator (TSO) expects wind power to cover $50 \%$ of annual Danish electricity consumption by 2025 [6], [7]. Large-scale integration of renewable energy sources is likely to introduce

Manuscript received November 27, 2013; revised March 29, 2014 and June 28, 2014; accepted July 14, 2014. Date of publication September 24, 2014; date of current version June 16, 2015. This work was supported in part by the Danish TSO Energinet.dk and in part by the Danish Council for Strategic research, grant agreement no. 11-116794. Paper no. TPWRS-01527-2013.

E. Dmitrova is with Energinet.dk, Fredericia, Denmark.

M. L. Wittrock, H. Jóhannsson, and A. H. Nielsen are with the Technical University of Denmark, Department of Electrical Engineering, Kgs. Lyngby 2800, Denmark.

Color versions of one or more of the figures in this paper are available online at http://ieeexplore.ieee.org.

Digital Object Identifier 10.1109/TPWRS.2014.2353693 faster and more severe fluctuations of the system operating point. In this regard, the computationally expensive offline approaches for stability and security assessment are foreseen to become insufficient for determining whether a rapidly fluctuating operating point is secure or not.

This problem has motivated development of methods which can assess system stability and security in real time. [8], [9] present a method that detects long-term voltage instability with sensitivities computed from wide-area snapshots, where full system observability is assumed. It does so by tracing computed sensitivities, using an algebraic system representation to ensure fast computation speed. [10], [11] present a method for long term voltage instability which utilizes Thevenin equivalents and local phasor measurements to calculate margins for maximal power transfer. For transient stability, [12] presents a method for early prediction of critical transient voltage sags caused by rotor swings, utilizing full system observability and the single machine infinite bus equivalent (SIME) method.

Of particular interest for this paper is the study of long term instability in the form of aperiodic small disturbance rotor angle (ASD) instability which has been explored in [13]. There, an efficient algorithm to compute generator power margins based on Thevenin impedance computations was shown to be suited to providing an early warning against ASD instability in real time. ASD stability is discussed in Section I-A and Section II-C

A common goal of these methods is to provide early warnings and increase situational awareness, but only to a lesser degree do they indicate which actions to take when an early warning is given in a critical situation. This paper presents a method which extends the results obtained in [13], to identify and evaluate suiteable countermeasures in the form of nodal admittance changes against emerging ASD instability.

The theoretical background is presented in Section II. The method is demonstrated on a simple 8-bus network in Section III. The method is then tested against a 464-bus model of the Western Denmark transmission system in Section IV.

The method is based on the results from [14]-[16] with the contribution of a test of a realistic model. This paper reflects the outcome of a Ph.D. dissertation and contributes by assembling the results into one coherent method [17].

Notation: Bold letters refer to matrices (e.g. $\boldsymbol{P}, \boldsymbol{v})$. Complex numbers are underlined (e.g. $\underline{Z}$ ).

\section{A. ASD Stability Boundaries}

In [13], a state-of-the-art method was developed to utilize real-time synchrophasor snapshots to quickly identify ASD stability margins of synchronous generators. The method uses algebraically derived expressions for stability boundaries, which enables assessment in linear time [18], [19]. It was shown that to ensure ASD stability, the injection impedance of a generator and the Thevenin impedance of the network, as seen from the 

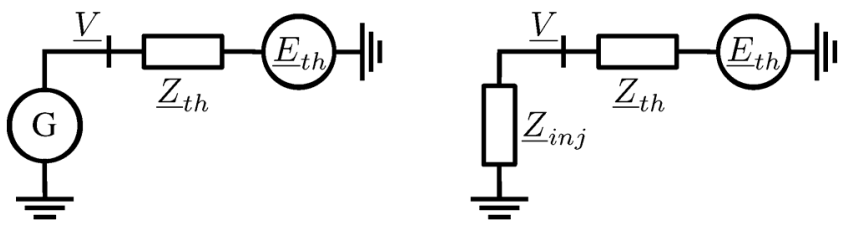

Fig. 1. Representing a generator in a two-bus system as an injection impedance.

point of constant steady-state voltage magnitude of the generator, must obey the condition

$$
Z_{i n j} \geq \frac{-Z_{t h} \sin \varphi_{i n j}}{\sin \phi_{t h}}
$$

Here $\underline{Z}_{i n j} \equiv Z_{i n j} \angle \varphi_{i n j}$ is the generator injection impedance with $Z_{i n j} \geq 0$ and $\underline{Z}_{t h} \equiv Z_{t h} \angle \phi_{t h}$ is the network Thevenin impedance with $Z_{t h} \geq 0$ as shown on Fig. 1 .

If (1) is violated, a small subsequent increase of the rotor angle will reduce the electrical output of the machine rather than increasing it. If the mechanical torque of the generator prime mover is not reduced, the electrical torque of the generator can no longer match the mechanical torque and the generator will accelerate, leading to loss of synchronism. For ASD instability, loss of synchronism can occur after ten of seconds and up to a few minutes. This mechanism is refered to in literature as aperidic rotor angle instability and has traditionally been treated using small-signal analysis.

The goal of this paper is to propose a method which determines and alter the power margins of the synchronous machines in the system. The injected generator power is

$$
P_{i n j}=\frac{V^{2}}{Z_{t h}} \cos \phi_{t h}-\frac{E_{t h} V}{Z_{t h}} \cos \left(\delta-\theta_{t h}+\phi_{t h}\right)
$$

where $\underline{E}_{t h} \equiv E_{t h} \angle \theta_{t h}$ is the Thevenin voltage and $\underline{V} \equiv V \angle \delta$ is the voltage at the point of constant steady-state voltage for the given generator, ensured by automatic voltage control. In this work $\delta$ is not the rotor angle, but the voltage angle at the point of constant voltage magnitude $V$. This is the terminal voltage when the generator is controlled by an AVR. When a generator is manually excited, the point of constant voltage moves behind the generator synchronous reactance $X_{d}$ and the value of $\delta$ will then reflect the rotor angle as well.

The maximal injectable power is

$$
P_{i n j, \max }=\frac{V^{2}}{Z_{t h}} \cos \phi_{t h}+\frac{E_{t h} V}{Z_{t h}}
$$

and a power margin can then be specified as

$$
\% \Delta P_{i n j}=\frac{P_{i n j, \max }-P_{i n j}}{P_{i n j, \max }} \cdot 100 \% .
$$

Using stability margins based on (1), the risk of ASD instability can be monitored in real time for every generator. This can be achieved by tracing $\underline{Z}_{t h}$ and $\underline{Z}_{i n j}$ for each generator in the grid if full observability can be provided by PMU's and state estimators [13].

If a violation is detected, stability could be restored by quickly rescheduling the output of the vulnerable generator. The early prevention method presented in this paper makes it possible to prevent emerging ASD instability by identifying the location

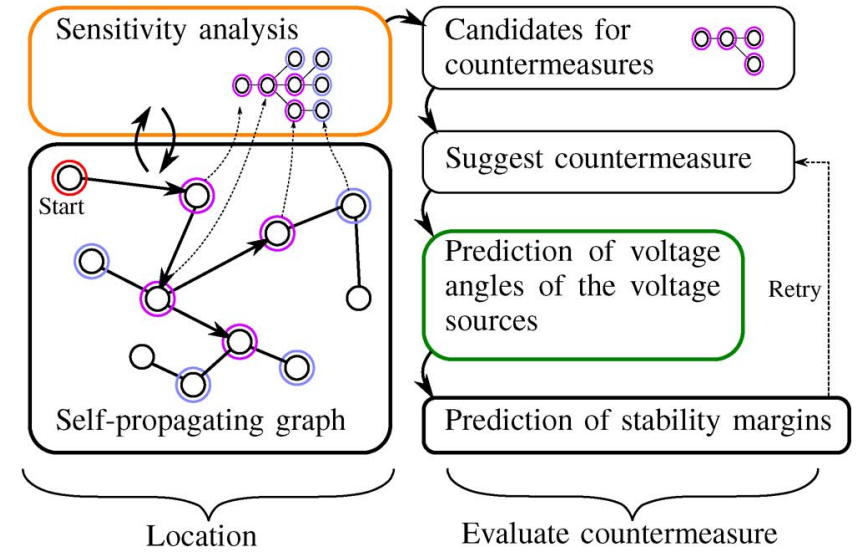

Fig. 2. Structure of the proposed early prevention method. Sensitivity analysis and self-propagating graphs are covered in Section II-A and voltage prediction is described in II-B and II-C.

and size of the necessary countermeasure in the form of nodal admittance change.

\section{Structure of the Early Prevention Method}

The purpose of the proposed method is to perform fast identification of effective countermeasures when a violation of the (1) is detected. The structure of the method and associated tasks is shown in Fig. 2. In the following sections, the theoretical basis for the developed method is described.

\section{A. Identification of Location for Countermeasure Application}

1) Sensitivity Analysis: On the basis of (1), a criterion which defines the grid nodes that have the highest impact on the stability of a given generator is proposed. The sensitivity of the Thevenin impedance to an alteration of a nodal admittance is used to indicate the potential efficiency of a given node to influence the generator ASD stability margin. A sensitivity matrix $\boldsymbol{S}_{K}$ is defined where each element of the matrix can be found according to

$$
S_{K_{k m}}=\frac{\partial\left(\frac{Z_{t h}}{\sin \phi_{t h}}\right)}{\partial Y_{m, m}} \frac{\partial K_{t h_{k}}}{\partial Y_{m, m}}
$$

where index $k$ refers to the considered generator and index $m$ corresponds to load nodes. The validity of using such sensitivities to identify nodes with considerable influence on the stability of a generator has been discussed in [14].

2) Self-Propagating Graphs: To reduce the number of nodes which need to be processed through the sensitivity analysis, an algorithm which reduces the number of nodes to be processed through sensitivity analysis has been proposed in [16]. This method is based on a self-propagating graph where the topologically nearest nodes are added to the graph in first order. The self-propagating graphs are used to select the nodes which will have their sensitivities computed. In each subsequent step, the nodes one vertex further compared to the previous step are added. The sensitivity (5) of each added node is computed, and each branch stops propagating when the sensitivity of the nodes on the previous step is below a preset $S_{\min }$. 


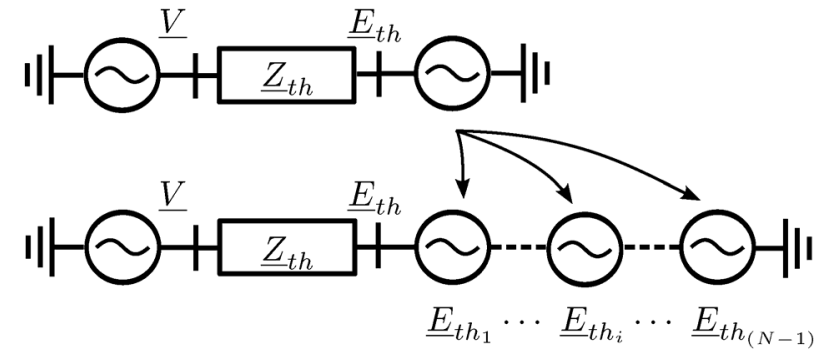

Fig. 3. Expansion of Thevenin equivalent for a system containing $N$ voltage sources.

This approach reduces the number of nodes which needs to be processed through sensitivity analysis by excluding nodes of minimal influence. The nodes added to the self propagating graph are placed in a prioritized list of candidates for countermeasure application. The prioritization is accomplished with respect to the sensitivity value and availability of control reserves. Reserves include reactive power compensation devices, energy storage, controllable loads and electrical vehicles.

After a prioritized list of candidates for countermeasure application is identified, an assessment of the size of countermeasure required to re-establish systems stability should be provided. The assessment should be computationally efficient to ensure near real-time operation. This excludes conventional time domain simulation based approaches, which are computationally intensive for large systems - especially when a system is approaching its stability boundaries where preventive countermeasures are urgently required. An approach for fast assessment is presented in Section II-B and Section II-C.

\section{B. Identification of the Thevenin Voltage Dependency on System Admittance Matrix Alteration}

From (1), one may notice that if it were possible to calculate $\underline{Z}_{i n j}$ and $\underline{Z}_{t h}$ for a post-countermeasure operating point, the stability assessment for that operating point could be carried out. An approach for determining $\underline{Z}_{t h}$ in real time using synchrophasor snapshots is presented in [20], while $\underline{Z}_{i n j}$ can be defined as

$$
\underline{Z}_{i n j}=\frac{\underline{V} \underline{Z}_{t h}}{\underline{E}_{t h}-\underline{V}}
$$

If it is possible to predict the value of $\underline{E}_{t h}$ and $\underline{V}$ after a countermeasure, it would be possible to assess if the countermeasure would lead the system to an attainable steady state by using (1) and (6).

To predict the value of $\underline{E}_{t h}$, the principle of superposition is utilized. The equivalent Thevenin voltage, represented as the sum of Thevenin voltage components induced by each voltage source in a grid, is shown in Fig. 3. Each of the $\underline{E}_{t h}$ components is defined by the value of the voltage measured at the point of constant steady state voltage magnitude and the topology of the grid. It has been shown in [15] that $\underline{E}_{t h_{i}}$ can be defined as a product of the voltage at the point of constant steady state

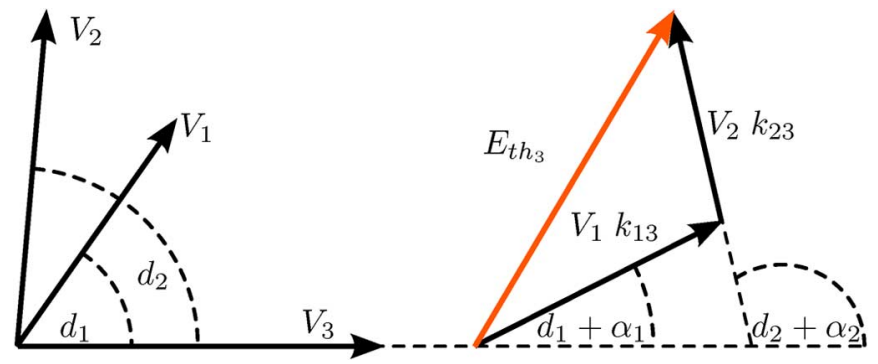

Fig. 4. Graphical illustration of the formulation of Thevenin voltage components using the GTC matrix elements $k_{j i}$.

voltage magnitude and a grid transformation coefficient (GTC) matrix

$$
\left(\begin{array}{c}
\underline{E}_{t h_{1}} \\
\underline{E}_{t h_{2}} \\
\vdots \\
\underline{E}_{t h_{N}}
\end{array}\right)=\underbrace{\left(\begin{array}{cccc}
0 & \underline{k}_{21} & \cdots & \underline{k}_{N 1} \\
\underline{k}_{12} & 0 & \cdots & \underline{k}_{N 2} \\
\vdots & \vdots & \ddots & \vdots \\
\underline{k}_{1 N} & \underline{k}_{2 N} & \cdots & 0
\end{array}\right)}_{G \boldsymbol{T C}} \cdot\left(\begin{array}{c}
\underline{V}_{1} \\
\underline{V}_{2} \\
\vdots \\
\underline{V}_{N}
\end{array}\right)
$$

where $\underline{k}_{i j} \equiv k_{i j} \angle \alpha_{i j}$.

The GTC matrix depends only on the grid topology and can be derived from the system admittance matrix. The GTC matrix elements $k_{j i}$ describe the contribution that a given voltage source $j$ has to the Thevenin voltage seen by generator $G_{i}$. The initial voltage vector, when seen as a component for the corresponding Thevenin voltage, changes angle and magnitude as seen in Fig. 4.

The sum of Thevenin voltage components form an equivalent Thevenin voltage seen by an arbitrary generator $M$ is

$$
\begin{aligned}
\underline{E}_{t h_{M}} & =\underline{V}_{1} \underline{k}_{1 M}+\underline{V}_{2} \underline{k}_{2 M}+\cdots+\underline{V}_{N} \underline{k}_{N M} \\
& =V_{1} e^{j \delta_{1}} k_{1 M} e^{j \alpha_{1}}+\cdots+V_{N} e^{j \delta_{N}} k_{N M} e^{j \alpha_{N}} \\
& =\sum_{\substack{i=1 \\
i \neq M}}^{N} V_{i} k_{i M} e^{j\left(\delta_{M}+\alpha_{M}\right)} .
\end{aligned}
$$

This representation makes it possible to assess how each of the Thevenin voltage components will be influenced by a disturbance in the grid. For this study in particular, the goal is to predefine how the equivalent Thevenin voltage will be influenced if a proposed countermeasure is applied. Separating the Thevenin voltages into components makes it possible to calculate their change corresponding to new operational conditions. As a result, the Thevenin voltage can be defined as a resulting vector of the sum of the components.

From (8), it is possible to calculate the new Thevenin voltage if, besides knowing GTCs $\underline{k}_{i}$, the value of voltage at the point of constant steady state voltage magnitude $\underline{V}_{i}$ is known. Since the early prevention method focuses on ASD stability, the magnitude of $\underline{V}_{i}$ under a new steady state operational point can be assumed to remain the same as under normal operation. It is thus required to determine what the angle of $\underline{V}_{i}$ under the new operating point would be. 


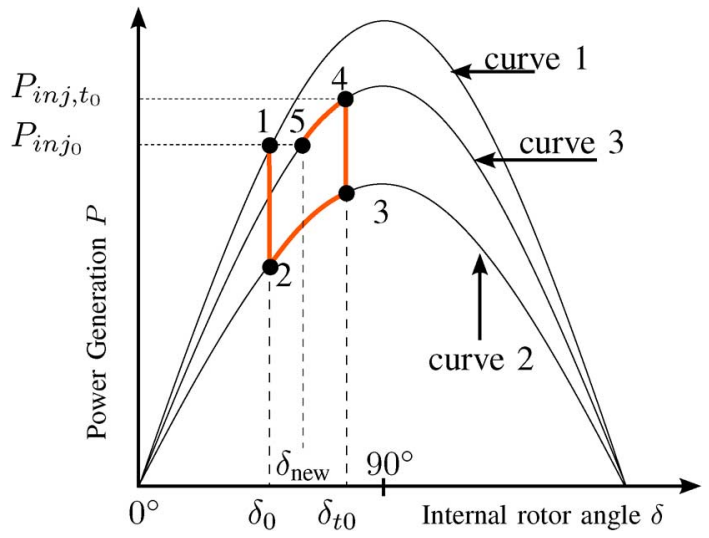

Fig. 5. Simplified trajectories of the rotor angle $\delta$ over $P \delta$ curves during initial steady state (curve 1), quasi steady state after a disturbance where the system gradually approaches the ASD stability boundary (curve 2) followed by an applied countermeasure (curve 3 ).

\section{Fast Assessment of the Effect of a Proposed Countermeasure}

This section presents an algorithm which enables an efficient prediction of the angles of $\underline{V}$ at the new operating point. Fig. 5 shows the $P \delta$ curve of a steady-state system subjected to a disturbance which leads to ASD instability and a subsequent countermeasure application. The five highlighted points on Fig. 5 are:

1) initial steady-state operating point (OP);

2) OP just after a disturbance;

3) OP where a countermeasure decision is taken;

4) OP just after the application of a countermeasure;

5) new stable steady-state OP.

In order to determine if an applied countermeasure would re-establish stability, the dynamics of the angle $\delta$ from point 3 to point 5 in Fig. 5 should be predicted for every generator in the grid. A method for such a prediction utilizing real-time synchrophasor system snapshots has been presented in [21].

The method uses the Newton algorithm to solve a system of $N-1$ nonlinear equations defined for the system, where $N$ is the number of voltage sources in the grid. Each of the system equations describes the active power flow for a two-bus system containing the voltage source in question and a Thevenin equivalent of the remaining system.

A graphical representation of the system is shown in Fig. 6 and is described by the system of equations

$$
P_{\text {gen }_{i}}-P_{i}\left(\delta_{i}\right)=0 \quad i=1,2, \ldots N-1
$$

which are to be solved for the voltage angles $\delta$ where

$$
P_{i}\left(\delta_{i}\right)=\frac{V_{i}^{2}}{Z_{t h_{i}}} \cos \phi_{t h_{i}}-\frac{E_{t h_{i}} V_{i}}{Z_{t h_{i}}} \cos \left(\delta_{i}-\theta_{i}+\phi_{t h_{i}}\right) .
$$

An iterative solution to determine the rotor angles $\boldsymbol{\delta}$ is now presented. First, an initial approximation of the voltage angles for the new steady state, suggested in [21], is given as

$$
\begin{aligned}
\delta_{k_{1}}= & \cos ^{-1}\left(\left(\frac{E_{t h_{t 0}} V}{Z_{t h_{t 0}}} \cos \left(\delta_{t 0}-\theta_{t 0}+\phi_{t h_{t 0}}\right)\right.\right. \\
& \left.\left.\quad+P_{i n j, t 0}-P_{i n j, 0}\right) \frac{Z_{t h_{t 0}}}{E_{t h_{t 0}} V}\right)+\theta_{t h_{t 0}}-\phi_{t h_{t 0}} .
\end{aligned}
$$

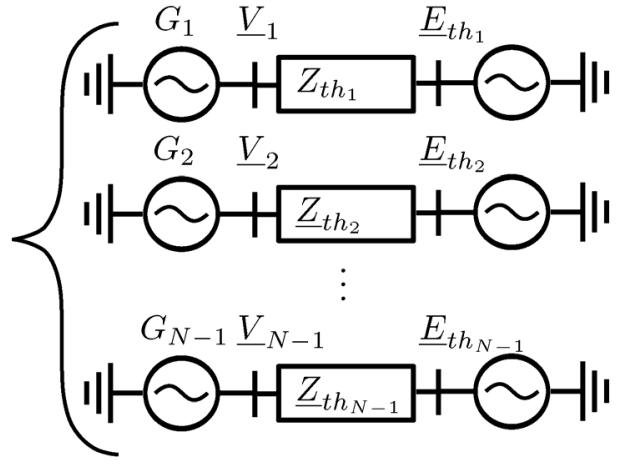

Fig. 6. Graphical representation of the system of equations each of which describes power flow in the grid containing given generator and corresponding Thevenin equivalent, substituting the remaining system.

The error can then be defined as

$$
\Delta P_{k}=P_{g e n}-P_{k}
$$

where $k$ is the iteration number. $\boldsymbol{\delta}$ is then updated to

$$
\delta_{k+1}=\delta_{k}+\Delta \delta_{k}
$$

with

$$
\Delta \boldsymbol{\delta}=\boldsymbol{J}^{-1} \Delta \boldsymbol{P} .
$$

Using the GTC matrix (7), $E_{t h}$ after the disturbance is

$$
\underline{\boldsymbol{E}}_{t h, k+1}=\boldsymbol{G T C} \cdot\left(\begin{array}{c}
V_{1} e^{j \delta_{1, k+1}} \\
V_{2} e^{j \delta_{2, k+1}} \\
\vdots \\
V_{N} e^{j \delta_{N, k+1}}
\end{array}\right) \text {. }
$$

The power injections $P_{i}\left(\delta_{i}\right)$ are then computed with (10) using (15) for $E_{t h}$ and the iterations continue until a sufficient accuracy is obtained. The Jacobian $\boldsymbol{J}$ used in (14) is

$$
\boldsymbol{J}(\boldsymbol{\delta})=\frac{\partial \boldsymbol{P}(\boldsymbol{\delta})}{\partial \boldsymbol{\delta}}
$$

where the diagonal elements are

$$
\frac{\partial P_{i}(\delta)}{\partial \delta_{i}}=\frac{E_{t h_{i}} V_{i}}{Z_{t h_{i}}} \sin \left(\delta_{i}-\theta_{t h_{i}}+\phi_{t h_{i}}\right)
$$

and the off-diagonal elements are

$$
\frac{\partial P_{i}(\delta)}{\partial \delta_{j}}=\frac{V_{i}}{Z_{t h_{i}}} \frac{V_{j} k_{j i}}{E_{t h_{i}}}\left(\operatorname{Re}\left(\underline{E}_{t h_{i}}\right) \sin \beta-\operatorname{Im}\left(\underline{E}_{t h_{i}}\right) \cos \beta\right)
$$

with $\beta \equiv\left(\delta_{i}-\theta_{t h_{i}}+\phi_{t h_{i}}+\delta_{j}+\alpha_{j i}\right)$. A derivation of (17) and (18) is found in [17].

The iterative process continues until a sufficient accuracy $\Delta \delta_{i}<\epsilon$ is achieved or the limit of iterations is exceeded due to non-convergence. Non-convergence indicates an unreachable steady state and hence ASD instability. If the iterative process converges, the obtained result is a solution for the new steadystate operational conditions corresponding to point 5 in Fig. 5.

The time-critical operations of the presented method are the computation of the sensitivities in (5) for the nodes selected by 


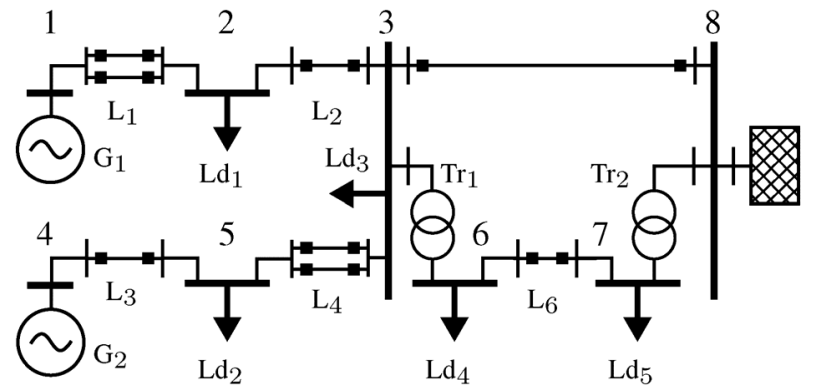

Fig. 7. An 8-bus test system used for validation of the early prevention method. Network data is available in [17].

the self-propagating graph, the computation of the GTC matrix used in (15) for the post-contingency network and the iterative solution of the system (8). Each sensitivity can be computed within milliseconds for large systems [19]. The GTC matrix can be computed efficiently using similar techniques if the algorithm presented in [19] is extended. In addition, the system (9) only contains $N$ equations, equal to the number of voltage-controlled nodes in the network. Approaches to simplify the computation of (9) have been explored in [21].

This provides a frame for which it is realistic to expect that a sufficient countermeasure can be suggested by the method within seconds. This makes the method suitable for fast online computations to counter emerging ASD instability or improve security margins. While the exact size of the countermeasure cannot be determined by the method without relying on an iterative process, the fast assessment makes it possible to evaluate many countermeasures quickly.

The stopping criterion of the self-propagating graph $s_{\min }$ is not well-defined at this point and further work will be required to find a suitable value for generic systems. Possible values of $s_{\min }$ and their influence have been explored in [16].

The method is able to treat ASD stability for disturbances that moves the system to a quasi-steady state and can thus manage both small and larger disturbances, provided the system survives the transient and an early warning from (1) is given early enough for a response. In the next sections, the early prevention method is demonstrated on a simple synthetic 8-bus network, and on a large-scale network model of the Western Denmark transmission system.

\section{Early Prevention Method ApPlied TO SYNTHETIC 8-BuS NETWORK}

A simple synthetic 8-bus network created to demonstrate the early prevention method is shown in Fig. 7.

$\mathrm{G}_{1}$ and $\mathrm{G}_{2}$ are manually excited, meaning that the point of constant steady-state voltage is behind the synchronous reactance $x_{d}$ of the generators. No AVR or power system stabilizers (PSS) are present in the system. The network is tuned to be heavily loaded and ASD instability can be provoked by increasing the load $\mathrm{Ld}_{1}$ by just $3 \%$ at a $t=5 \mathrm{~s}$, which leads to instability at $t \approx 38 \mathrm{~s}$ as shown in Fig. 8 . The ASD stability boundary (1) is crossed at $t=18.6 \mathrm{~s}$.

The early warning triggers a search for appropriate nodes for applying countermeasures. A self-propagating graph, rooted at

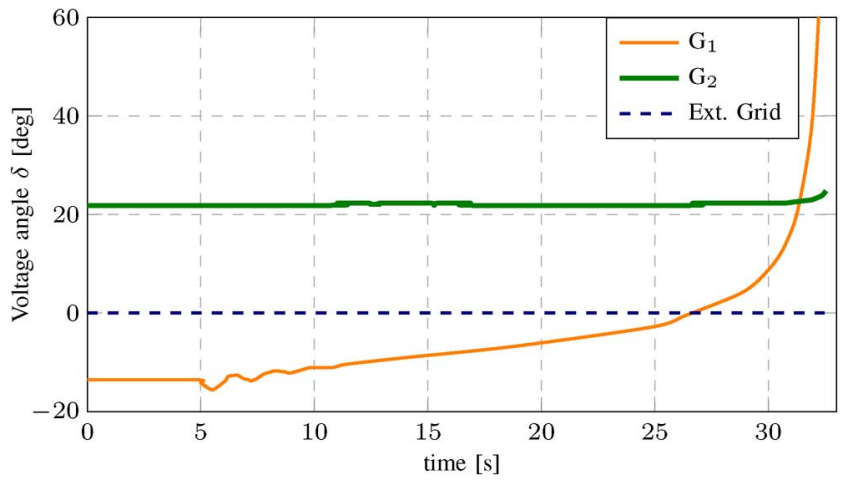

Fig. 8. Voltage angles of generator $\mathrm{G}_{1}, \mathrm{G}_{2}$ and the external grid. Instability is caused by a load increase of $\operatorname{Ld}_{1}$ by $3 \%$.
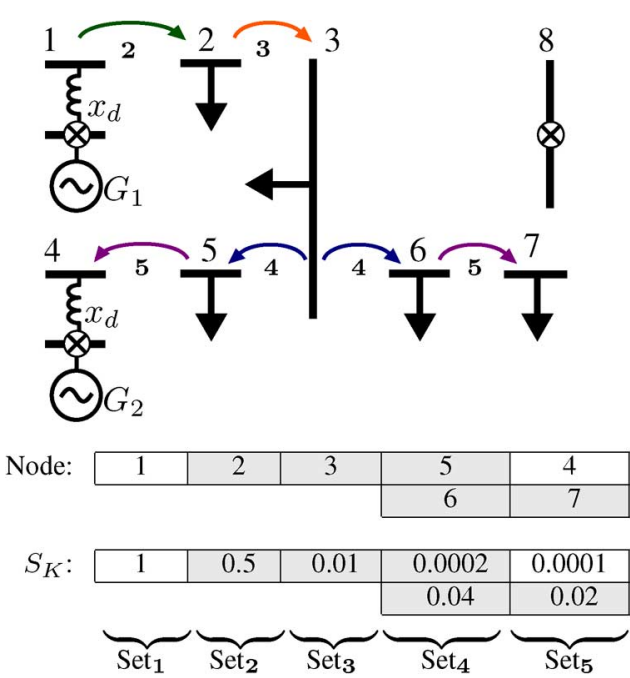

Fig. 9. Sensitivity values $S_{K}$ corresponding to the nodes added to the selfpropagating graph. Bus 8 and the nodes behind $x_{d}$ have voltage control and are not considered by the graph. Gray cells indicate nodes with available control reserves.

TABLE I

Prediction AND Simulation of Power Margin

\begin{tabular}{ccccc|c}
\hline Gen & $\delta_{\text {pred }}$ & $\delta_{\text {sim }}$ & $P_{\text {mar,pred }}$ & $P_{\text {mar,sim }}$ & $N_{\text {itr }}$ \\
\hline$G_{1}$ & $19.81^{\circ}$ & $19.84^{\circ}$ & $0.63 \%$ & $0.63 \%$ & 27 \\
$G_{2}$ & $50.16^{\circ}$ & $50.19^{\circ}$ & $0.64 \%$ & $0.63 \%$ & 27 \\
\hline
\end{tabular}

the vulnerable generator $G_{1}$ at bus 1, propagates through neighboring nodes as seen in Fig. 9 and the sensitivity for each visited node is calculated. This identifies bus 2 as the most suited node for a countermeasure. This is expected, since node 2 is the nearest node with available control resources.

Following the identification of the optimal nodes for stability improvement, a countermeasure in the form of reactive power injection at bus 2 is attempted. The nodal admittance $Y_{22}$ is decreased by $0.005 j$ per unit. The effectiveness of the countermeasure is evaluated according to (12)-(18) and the stopping criterion of the obtained solution was set to $\Delta \delta_{i}<10^{-5} \mathrm{deg}$. The evaluation can be seen in Table I, where the results obtained using the fast assessment method are compared with those obtained using a time domain simulation, where $P_{\mathrm{mar}}$ is the active power stability margin calculated using (4). 


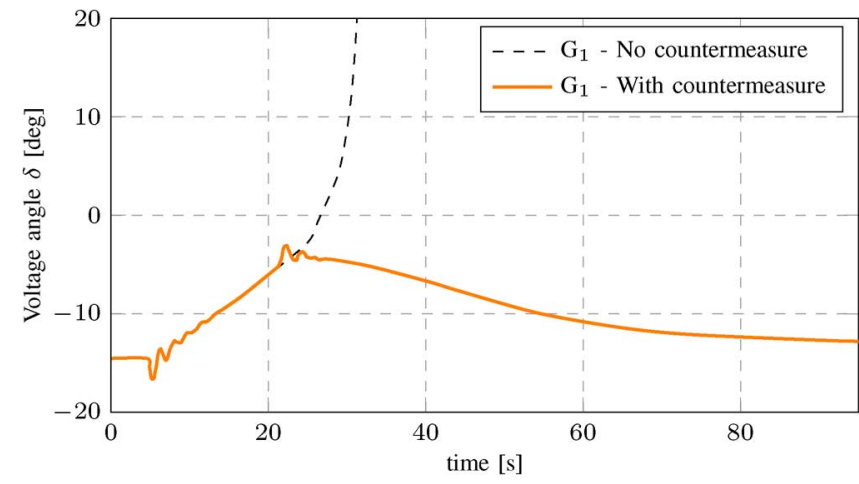

Fig. 10. Voltage angles of $\mathrm{G}_{1}$ and the external grid when applying countermeasure at boundary detection.

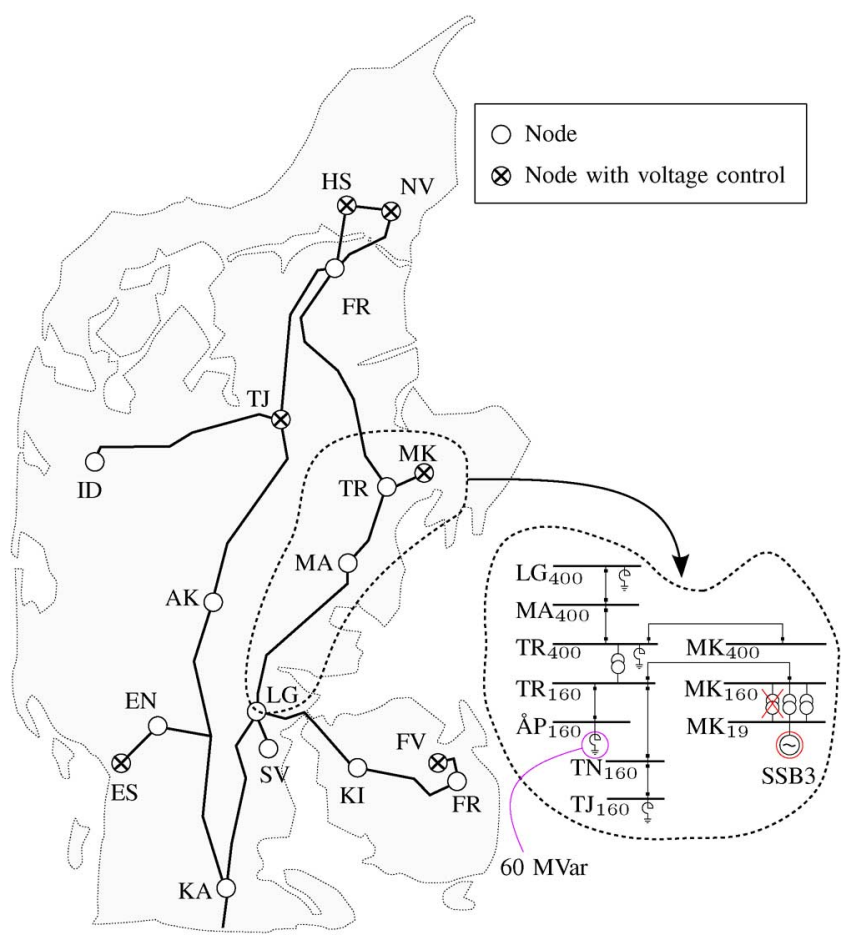

Fig. 11. 400-kV grid model of the Western Denmark Transmission system used in this study. A detailed view of a selected area is included with contingency and countermeasure shown.

Fig. 10 shows the change of the voltage angles at the voltage controlled buses in the system when the suggested countermeasure is applied at $t=22 \mathrm{~s}$. It is seen that the predicted and the simulated angles are in good agreement with each other. As expected, the countermeasure stabilizes the system as it was possible to find an attainable steady-state voltage. Had the iterations not converged, the countermeasure would not have been sufficient.

\section{Large Scale Test on Model OF THE WESTERN DENMARK SYSTEM}

The method was tested on a model acquired from the Danish TSO Energinet.dk, which represents the transmission system in Western Denmark shown in Fig. 11. The model is used by the
TSO for dynamic security studies. The Western Denmark transmission system is operated at $400 \mathrm{kV}$ and $160 \mathrm{kV}$. The distribution system runs at $60 \mathrm{kV}, 10 \mathrm{kV}$ and finally at $0.4 \mathrm{kV}$ at households. Six HVDC interconnections are operated (three to Norway, two to Sweden and one to Zealand), as well as an AC interconnection to Germany. The loads in the model are aggregated at $60 \mathrm{kV}$ and $160 \mathrm{kV}$. The model is set for a total load of $3.7 \mathrm{GW}$. The installed capacity of the central power plants is 1.8 $\mathrm{GW}$, while local combined heat and power plants account for 2 GW. Wind power accounts for $2.9 \mathrm{GW}$ in the case study. System voltage is maintained by tap changing transformers, shunt compensators and voltage control by the main power stations as shown in Fig. 11.

The model is a DIgSILENT PowerFactory model. It contains 464 nodes, 162 lines, 377 2-winding transformers, 103 -winding transformers, 56 asynchronous machines, 150 synchronous machines, 68 shunt units and 29 static generators. Wind turbines are modeled as asynchronous machines and static generators with dynamic models written in the DSL language of PowerFactory. The models for wind turbines and local generation regulates according to constant power factor. Interconnections are modeled as static loads.

\section{A. System Data Acquisition}

The early prevention method was implemented in MatLab and used power system simulation software to provide synthetic synchrophasor snapshots. With the Energinet.dk model, manual data import was not feasible and automatic software had to be developed to analyze the network and extract the required data, grid parameters and system snapshots. Such a tool was available and designed exactly for the task of extracting data from power system simulation software and importing it to MatLab [22].

The tool made it possible to acquire the data in a well-structured form which could be parsed to previously developed MatLab tools. The tool has previously been used to replicate the results obtained in [8] using a PowerFactory model [22].

\section{B. Western Denmark Power System Study Case}

To test the developed algorithms, it is necessary to alter the model in such a manner that ASD instability is provoked.

In the original model, all voltage-regulated generators have AVRs and PSSs with the initial operating point being far from over-excitation. This results in large stability margins and a secure system. To find the most vulnerable generator, the stability boundaries from (1) were examined when the generators were manually excited.

Generator SSB3 would have the lowest stability boundary with $P_{\mathrm{mar}}=0.35 \%$ if its excitation system is overruled to manual mode. With the AVR and PSS disabled, generator SSB3 becomes vulnerable to ASD instability.

Next, a contingency, triggering instability, is to be found. The 455 MWA step-up transformer, which connects generator SSB3 to the grid, was replaced with three parallel transformers each rated 260 MVA to provide a better opportunity to initiate instability. The contingency considered is the trip of one of these transformers. The transformer trip is shown on Fig. 11.

Fig. 12 shows the result of a transformer trip when generator SSB3 is set to manual excitation. The described contingencies provoke ASD instability providing a suitable case study. 


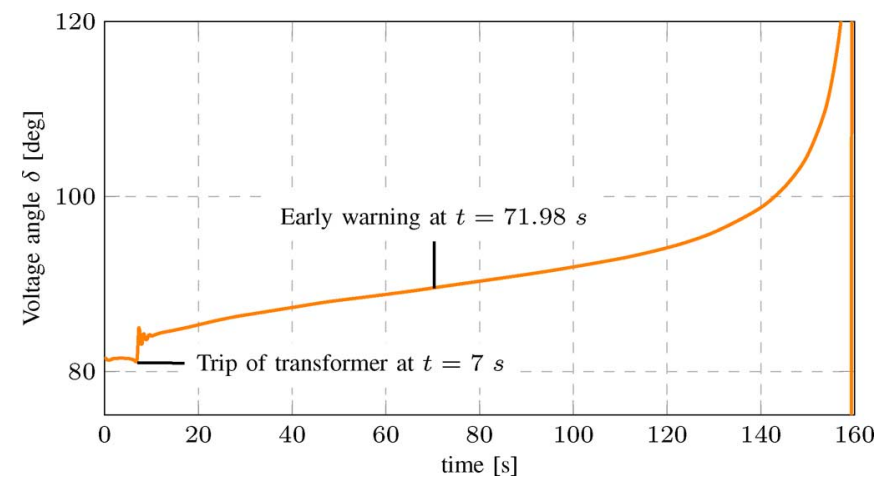

Fig. 12. Voltage angle of generator SSB3. The generator loses synchronism in about $150 \mathrm{~s}$ after the contingency.

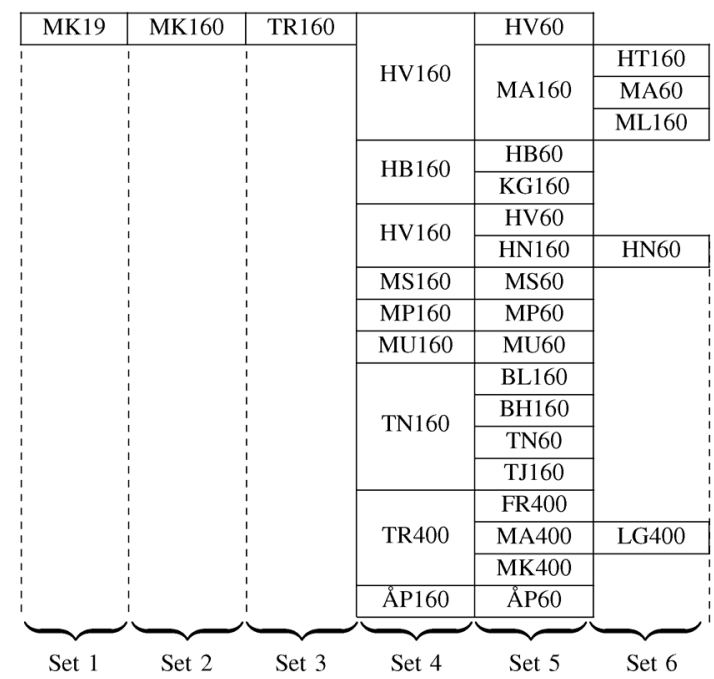

Fig. 13. Sets of nodes sequentially added to the self-propagating graph.

\section{Early Prevention Method Demonstration}

Following the procedures outlined in Sections II and III, the first step is to evaluate and identify the optimal locations for countermeasure application. A self-propagating graph rooted at the vulnerable generator SSB3 finds a set of nodes shown in Fig. 13.

The sensitivities $S_{K}$ for the added nodes are shown in Fig. 14. Based on the obtained sensitivities and availability of control reserves, the node $\AA \mathrm{P} 160$ is chosen as a first candidate for a countermeasure. The control reserve available at node $\AA \mathrm{P} 160$ is a single step 60 MVar inductive reactor $\AA \mathrm{P} 160$ - ZL1. Disconnecting this unit would decrease the system impedance and can be considered as a countermeasure.

To evaluate the effect of this countermeasure, the voltage angles are predicted using the fast assessment procedure described in II-C. The first approximation of the voltage angles at the settling point (point 5 in Fig. 5), corresponding to the new operating point for all generators in the system, is presented in Table II. The final voltage angles are computed using the iterative process (12)-(15), described in Section II-C. The iterations continue until $\Delta \delta<1^{\circ}$ or the maximum number of iterations $N_{i t r}$ is exceeded. Table III shows the results obtained by the

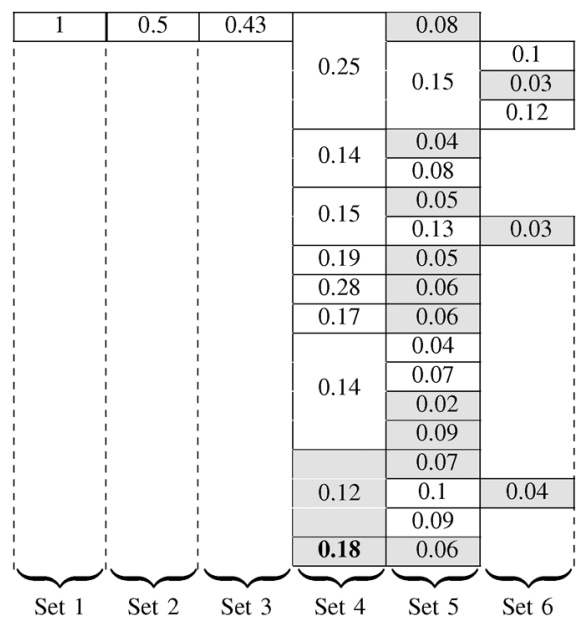

Fig. 14. Gray cells mark nodes with available control resources.

TABLE II

First APPRoXimation of COMPUTEd Voltage ANGLES SUBSEQUENT TO COUNTERMEASURE APPLICATION (IN DEG)

\begin{tabular}{lcccccc}
\hline Gen & BDOR & BRUN & FYB7 & KIEL & SSB4 & NJB3 \\
\hline$\delta\left[^{\circ}\right]$ & 178.30 & -1.97 & 8.24 & -7.87 & 9.07 & 6.00 \\
\hline
\end{tabular}

\begin{tabular}{cccccc}
\hline Gen & TJE & VHA & ESB3 & SSB3 & UCTE \\
\hline$\delta\left[^{\circ}\right]$ & -0.62 & 2.52 & 20.05 & 81.51 & 0 \\
\hline
\end{tabular}

TABLE III

Prediction of Power Margins for 464-Bus Model

\begin{tabular}{|c|c|c|c|c|c|}
\hline Gen & $\begin{array}{c}\delta \\
\text { Pre, }\left[^{\circ}\right] \\
\end{array}$ & $\begin{array}{c}\delta \\
\operatorname{Sim},\left[^{\circ}\right] \\
\end{array}$ & $\begin{array}{l}\Delta P_{i n j} \\
\text { Pre, } \% \\
\end{array}$ & $\begin{array}{l}\Delta P_{i n j} \\
\text { Sim, \% } \\
\end{array}$ & $N_{i t r}$ \\
\hline BDOR & 178.18 & 178.15 & 100.00 & 100.00 & \multirow{10}{*}{14} \\
\hline BRUN & -2.08 & -2.12 & 100.00 & 100.00 & \\
\hline FYB7 & 7.81 & 7.78 & 80.55 & 80.54 & \\
\hline KIEL & -8.06 & -8.09 & 100.00 & 100.00 & \\
\hline SSB4 & 8.52 & 8.48 & 84.06 & 84.05 & \\
\hline NJB3 & 5.44 & 5.39 & 85.45 & 85.43 & \\
\hline TJE & -1.18 & -1.22 & 100.00 & 99.99 & \\
\hline VHA & 1.92 & 1.88 & 100.00 & 100.00 & \\
\hline ESB3 & 19.63 & 19.59 & 77.30 & 77.29 & \\
\hline SSB3 & 85.10 & 85.89 & 0.47 & 0.32 & \\
\hline
\end{tabular}

early prevention method compared with a time domain simulation. As a solution was attainable, the suggested countermeasure is expected to prevent the emerging instability.

Fig. 15 shows the change of rotor angle for the critical generator SSB3 when the suggested countermeasure is applied. As can be seen from Fig. 15, a new steady state is reached $120 \mathrm{~s}$ after the countermeasure. Fig. 16 shows the $P \delta$ trajectory for the study with respect to the synchronous reactance of generator SSB3 when the countermeasure is applied. Note that the generator mechanical input is $350 \mathrm{MW}$. After a short transient period, the angle begins to steadily increase towards the limit. Fig. 17 shows the generator response to the countermeasure. Even when beyond the point of maximum power transfer, the 


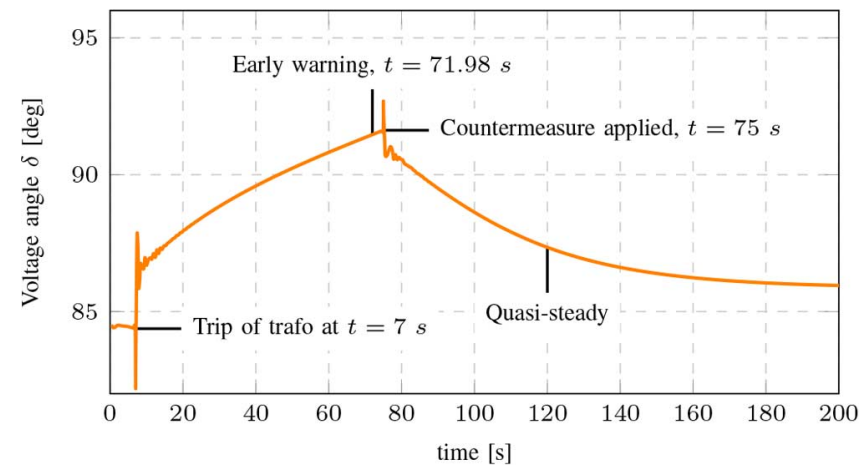

Fig. 15. Voltage angle of generator SSB3 after applying a countermeasure to restore stability.

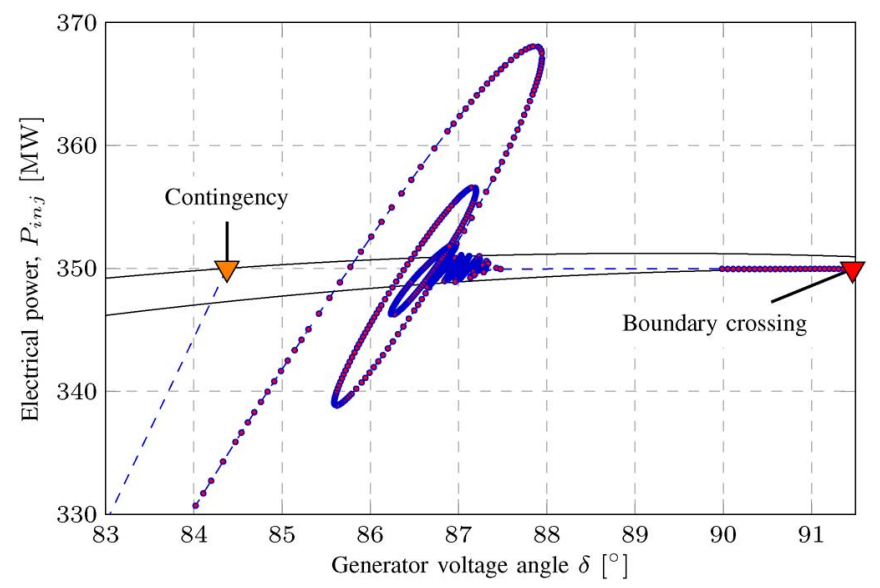

Fig. 16. Trajectory of $\delta$ of generator SSB3 after the contingency with respect to the synchronous reactance $X_{d}$.

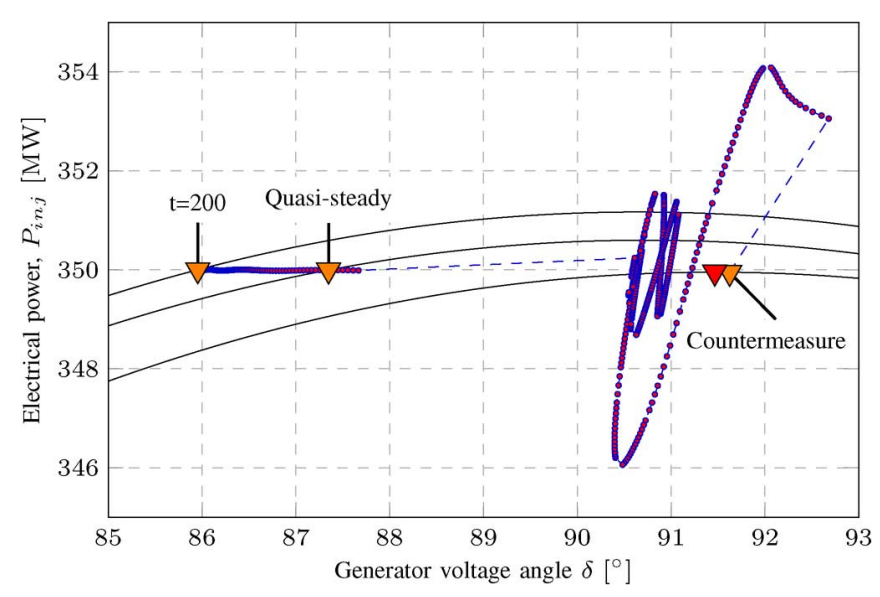

Fig. 17. Trajectory of $\delta$ of generator SSB3 when applying countermeasure.

voltage angle starts declining and reaches a new steady-state after the initial dynamic response of the angle. It can be argued that the system enters a safe state shortly after the countermeasure, which is typical for ASD stability. For illustrative purposes, the countermeasure was applied $3 \mathrm{~s}$ after the early warning to account for countermeasure assessment calculations and signal propagation time to the equipment. The trajectory of the angle is shown in Fig. 18 in a simplified version similar to Fig. 5. Here the mechanism of ASD instability can be seen,

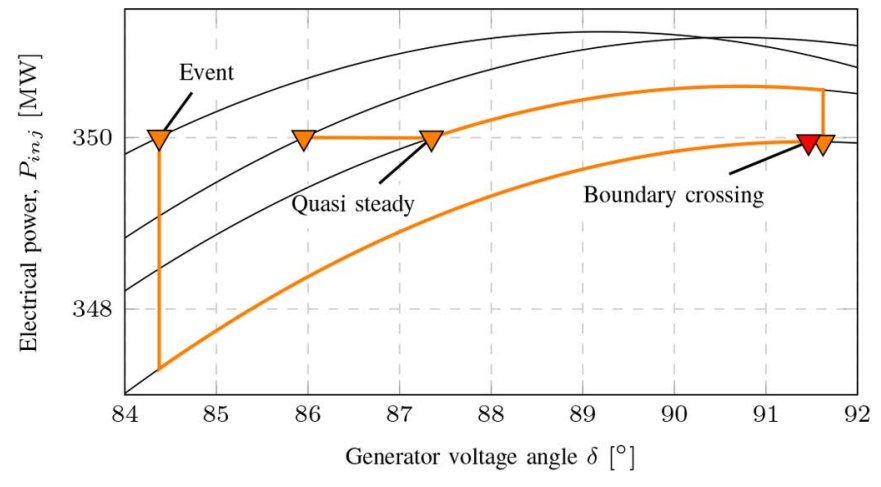

Fig. 18. $P \delta$ curves of generator SSB3 with respect to synchronous reactance $X_{d}$ and simplified trajectory of the angle similar to Fig. 5.

where the generator just barely is unable to match its mechanical torque with its electrical torque until the countermeasure is engaged.

As can be seen from Table III, the voltage angles are accurately predicted by the method. The presented case study of the Western Denmark system demonstrates the capability of the early prevention method to evaluate a countermeasure's ability to re-establish stability and predict the resulting stability margins for the new steady state.

\section{CONCLUSION AND DisCussion}

This paper presented an early prevention method which exploits full system observability provided by real-time synchrophasor measurements and state estimation to detect emerging aperiodic small disturbance rotor angle (ASD) instability. When ASD instability is detected, the method identifies candidates for countermeasure by calculating the sensitivities of ASD stability indicators for load nodes. Only the most significant nodes have their sensitivities calculated as they are visited by a self-propagating graph, rooted at the vulnerable generator. The method relies on full system observability, which is expected to be available in the future power system.

The GTC matrix was introduced, providing a relation between a change of impedance and the resulting Thevenin voltage as seen from a corresponding generator. The effect of a countermeasure could then be predicted with an iterative algorithm which predicts the steady state voltages of the system after an applied countermeasure. The steady state response of a countermeasure could then be predicted and the new stability margins could be assessed to determine if the countermeasure was sufficient to re-establish ASD stability.

The method was validated against a simple synthetic 8-bus network and against a 464-bus model of the Western Denmark transmission system. For the latter case, the method computed the sensitivities of 34 busses visited by the graph, from which a suitable location for a countermeasure could be found. Fourteen iterations of the numerical solution determined the result of the suggested countermeasure correctly.

The presented results show that the developed method can be applied to efficiently find suitable countermeasures against emerging ASD instability for networks where wide-area monitoring systems provide full observability. Furthermore, many of the principles and concepts presented in this work may be expanded to include other stability mechanisms and to consider 
exploiting several nodes simultaneously for applying countermeasures. Further research has already been funded to look deeper into these topics.

\section{ACKNOWLEDGMENT}

The authors would like to thank Energinet.dk for providing the model of the Western Denmark power system.

\section{REFERENCES}

[1] Y. Makarov, V. Reshetov, V. Stroev, and N. Voropai, "Blackouts in north america and europe: Analysis and generalization," in Proc. IEEE St. Petersburg PowerTech 2005, 2005, pp. 1-7.

[2] B. Liscouski and W. Elliot, Final Report on the August 14, 2003 Blackout in the United States and Canada: Causes and Recommendations, A report to the U.S. Department of Energy, 2004, Tech. Rep.

[3] F. Vandenberghe, E. Grebe, D. Klaar, K. Kleinekorte, J.-M. Rodriguez, H. Erven, H. Laffaye, C. Sabelli, F. Kropec, T. Tillwicks, L. Tassan, S. Callewaert, M. Mandozzi, K. Imhof, N. Janssens, and O. Bronckart, Final Report of the Investigation Committee on the 28 september 2003 blackout in Italy, UCTE, Apr. 2004, Tech. Rep.

[4] S. Larsson and E. Ek, "The black-out in southern Sweden and eastern Denmark, September 23, 2003," in Proc. IEEE Power Engineering Society General Meeting, 2004, vol. 2, pp. 1668-1672.

[5] Task Force on Discrete Supplementary Controls of the Dynamic System Performance Working Group of the IEEE Power System Engineering Committee, "Description of discrete supplementary controls for stability," IEEE Trans. Power App. Syst., vol. PAS-97, no. 1, pp. 149-165, Jan. 1978.

[6] P. Sørensen, M. Togeby, T. Ackermann, D. Chandrashekhara, J. Horstmann, H. Jóhannsson, A. Nielsen, P. Nyeng, T. Rasmussen, and Z. Xu. et al., Ecogrid. dk phase 1 wp4 Report: New Measures for Integration of Large Scale Renewable Energy, Danmarks Tekniske Universitet, Risø Nationallaboratoriet for Bæredygtig Energi, 2008, Tech. Rep.

[7] M. Trong, K. Nørregaard, J. Østergaard, P. Bach, M. Lind, P. Sørensen, B. Tennbakk, M. Togeby, and T. Ackermann, Ecogrid. dk Phase i Summary Report: Steps Towards a Danish Power System With 50\% Wind Energy, Energinet.dk, 2009, Tech. Rep.

[8] M. Glavic and T. Van Cutsem, "Wide-area detection of voltage instability from synchronized phasor measurements. part 1: Principle," IEEE Trans. Power Syst., vol. 24, no. 3, pp. 1408-1416, Aug. 2009.

[9] M. Glavic and T. Van Cutsem, "Wide-area detection of voltage instability from synchronized phasor measurements. part ii: Simulation results," IEEE Trans. Power Syst., vol. 24, no. 3, pp. 1417-1425, Aug. 2009.

[10] S. Corsi and G. Taranto, "A real-time voltage instability identification algorithm based on local phasor measurements," IEEE Trans. Power Syst., vol. 23, no. 3, pp. 1271-1279, Aug. 2008.

[11] G. N. Taranto, C. Oyarce, and S. Corsi, "Further investigations on phasor measurement-based algorithm utilized for voltage instability awareness," in Proc. 2013 IREP Symp. Bulk Power System Dynamics and Control-IX Optimization, Security and Control of the Emerging Power Grid, Aug. 2013, pp. 1-8, IEEE.

[12] T. Weckesser, H. Jóhannsson, and T. Van Cutsem, "Early prediction of transient voltage sags caused by rotor swings," in Proc. 2014 IEEE PES General Meeting, 2014

[13] H. Jóhannsson, A. H. Nielsen, and J. Østergaard, "Wide-area assessment of aperiodic small signal rotor angle stability in real-time," IEEE Tran. Power Syst., vol. 28, no. 4, pp. 4545-4557, Nov. 2013.

[14] E. Dmitrova, H. Jóhannsson, and A. H. Nielsen, "Early prevention of instability-search for optimal grid nodes for applying countermeasures," in Proc. Int. Conf. Environment and Electrical Engineering EEEIC, IEEE Venice, 2012.

[15] E. Dmitrova, H. Jóhannsson, and A. H. Nielsen, "Assessment of the impact that individual voltage source has on a generators stability," in Proc. 10th Int. Power and Energy Conf. IPEC, Ho Chi Minh, Vietnam, Dec. 2012.

[16] E. Dmitrova, H. Jóhannsson, and A. H. Nielsen, "Early prevention of instability-use of self propagating graph for the fast search for optimal grid nodes to apply countermeasures," in Proc. IEEE PowerTech Conf. 2013, Grenoble, France, Jun. 2013.
[17] E. Dmitrova, "Early prevention method for power systems instability," Ph.D. dissertation, Tech. Univ. Denmark, Kgs. Lyngby, Denmark, 2013.

[18] H. Jóhannsson, A. Nielsen, and J. Østergaard, "Identification of critical transmission limits in injection impedance plane," Int. J. Elect. Power Energy Syst., vol. 43, no. 1, 2012.

[19] S. Sommer and H. Jóhannsson, "Real-time Thevenin impedance computations," in Proc. 2013 IEEE ISGT Washington Conf., Feb. 2013.

[20] H. Jóhannsson, R. Garcia-Valle, J. Weckesser, A. Nielsen, and J. Østergaard, "Real-time stability assessment based on synchrophasors," in Proc. 2011 IEEE Trondheim PowerTech, 2011, pp. 1-8.

[21] E. Dmitrova, H. Jóhannsson, and A. H. Nielsen, "Fast assessment of the effect of preventive wide area emergency control," in Proc. 4th IEEE Eur. Innovative Smart Grid Technologies (ISGT) Conf., Copenhagen, Denmark, Oct. 2013.

[22] M. L. Wittrock and H. Jóhannsson, "An implementation and test platform for wide area stability assessment methods," in Proc. IEEE Power Engineering Society ISGT Conf., Copenhagen, Denmark, 2013.

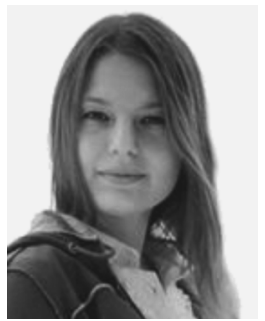

modern power systems.
Evgenia Dmitrova received the B.S. degree from Moscow Power Engineering Institute, Russia, in 2007, the M.S. degree from Lappeenranta University of Technology, Finland, in 2009, the Specialist degree from Moscow Power Engineering Institute, Russia, in 2010, and the Ph.D. degree from the Technical University of Denmark in 2013.

She is currently a power engineer at the system operation department of the Danish TSO Energinet.dk. Her research interests lie within the area of real-time stability monitoring and preventive control for

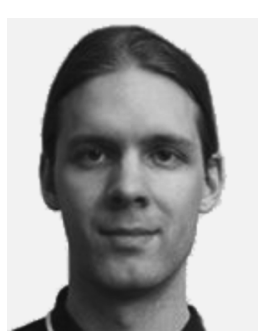

Martin Lindholm Wittrock (S'12) received the M.Sc degree in electrical engineering from the Technical University of Denmark in 2012. He is currently pursuing the $\mathrm{Ph} . \mathrm{D}$. degree in wide area prosumption control at Centre for Electric Power and Energy, Department of Electrical Engineering, Technical University of Denmark.

His research interests lies in power system stability and security and wide-area monitoring systems.

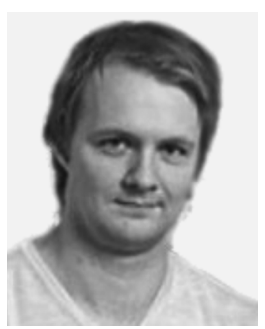

Hjörtur Jóhannsson (M’11) received the M.Sc. and the $\mathrm{Ph} . \mathrm{D}$. degrees in electrical engineering from Technical University of Denmark in 2007 and 2011, respectively.

$\mathrm{He}$ is currently an Assistant Professor at Center for Electric Power and Energy (CEE), Department of Electrical Engineering, Technical University of Denmark. His research interests concern the development of methods that provide an early warning for instability in electric power systems, and power systems dynamics, stability, and control.

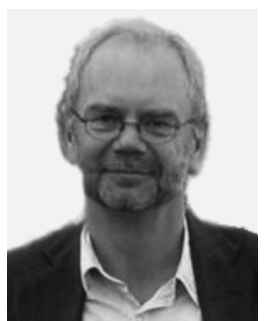

Arne Hejde Nielsen (SM'08) is an Associate Professor at Center for Electric Power and Energy (CEE), Department of Electrical Engineering, Technical University of Denmark. He has 30 years of experience in electric power engineering; the first years were from ASEA AB, Central Research and Development Department, Sweden, working with measurement technology, motor design, and control. During the latest 10 years, his focus has been on electric power systems, especially on implementation of renewable energy sources in the power system. 